\title{
A multichannel computer-driven system to raise aquatic embryos under selectable hypoxic conditions
}

This article was published in the following Dove Press journal: Hypoxia

\section{Sanjeeva Metikala ${ }^{1,2, *}$ \\ Herbert Neuhaus ${ }^{1, *}$ \\ Thomas Hollemann' \\ 'Department of Medical Molecular Biology, University Halle-Wittenberg, Institute for Physiological Chemistry, Halle, Germany; ${ }^{2}$ Division of Developmental Biology, Cincinnati Children's Hospital Medical Center, Cincinnati, $\mathrm{OH}$, USA}

*These authors contributed equally to this work
Correspondence: Thomas Hollemann University Halle-Wittenberg, Institute for Physiological Chemistry, Hollystrasse I, 06 I I 4 Halle, Germany

Tel +49345 5573668

Email thomas.hollemann@medizin.unihalle.de

\begin{abstract}
The formation of a functional cardiovascular system is an essential step in the early vertebrate embryo. Nevertheless, the effect of hypoxia on the developmental program of organisms was studied rarely. In particular, this holds true for vertebrate embryos that depend on a functional placenta for proper development and had not been studied in this respect due to the obvious limitation. We established a protocol to culture aquatic embryos, which enabled us to culture a high number of Xenopus embryos until tadpole stage under defined hypoxic conditions in four hypoxia chambers simultaneously, employing a computerized system. In general, our results show that hypoxia results in delayed development and, in particular, we could show that oxygen availability was most crucial during gastrulation and organogenesis (early tailbud) phases during embryonic development of Xenopus laevis.
\end{abstract}

Keywords: Xenopus, hypoxia, vasculogenesis, culture conditions, er71, ami

\section{Introduction}

The effects of hypoxia on organisms were widely studied in the context of tumor microenvironment and neovascularization. These studies are mostly based on cultured cells and adult organisms but do not focus on vertebrate vasculogenesis taking place during early embryogenesis. To study the effect of hypoxia, a panel of tools was developed. In cell cultures, commercially available hypoxia chambers were used. ${ }^{1,2}$ Completely home-made, tightly sealed Lucite chambers were used in early experiments using rodents. ${ }^{3}$ Similar designs were also used by other groups with each of the systems having their own set of application-oriented advantages and limitations. ${ }^{4}$

Recently, the interest in studying the effects of hypoxia on embryogenesis increased. Several reports, using different aquatic model organisms, analyzed the effect of hypoxia on various cellular and morphological processes during early development. In painted turtles, Chrysemys picta, the influence of hypoxic conditions on body weight was analyzed; 5 in mussel, Mytilus coruscus, the influence of hypoxic conditions on hemocyte mortality was analyzed; ${ }^{6}$ in the estuarine killifish, Fundulus heteroclitus, the influence of hypoxic conditions on mitochondrial physiology was analyzed; ${ }^{7}$ and in the blue crab, Callinectes sapidus, the influence of hypoxic conditions on metabolic rate was analyzed. ${ }^{8}$

Similar studies were also performed in adult vertebrates to study hypoxia-induced cerebral angiogenesis in mouse cortex ${ }^{9}$ and hypoxia-induced neovascularization in zebrafish retina. ${ }^{10}$ Although a detailed protocol for culturing zebrafish under hypoxia 
was available, ${ }^{11}$ it was limited to a single chamber and allows culturing embryos in less numbers only. To test the effect of hypoxia in a series of different oxygen levels, utilizing a single-tank system would be very time consuming. This defines the need for a system allowing to regulate multiple hypoxia chambers at the same time. Hence, we developed a protocol to maintain four tanks at different hypoxic levels simultaneously with 100 aquatic embryos in each tank.

In our previous study, ${ }^{12}$ we employed embryos of the African clawed frog, Xenopus laevis, to study the effect of hypoxia on the formation of cardiovascular system. $X$. laevis is an excellent model to study the effect of hypoxia as the embryos develop extrauterine, which facilitates the handling and culturing in hypoxic chambers. Its evolutionary closeness to humans was advantageous in studying various developmental processes.

In the above study, we exposed the embryos to hypoxic conditions after the first cleavage of the embryo (Nieuwkoop $\&$ Faber [NF] stage 2) and incubated further for 3-5 days until the embryos reached NF stage 37 and then harvested. However, the definitive phase that was most sensitive to hypoxia during development still remains to be elucidated. In the current study, we investigated the developmental phase during which the embryos were most vulnerable to reduced oxygen levels, studied the effect of hypoxia on embryonic growth and viability, and provided a detailed protocol to culture $X$. laevis embryos under hypoxic conditions.

\section{Materials and methods Experimental model}

Production and rearing of $X$. laevis embryos were done as described earlier, ${ }^{13}$ and the embryos were staged according to Nieuwkoop and Faber. ${ }^{14}$ All procedures were in accordance with the guidelines from Directive 2010/63/EU of the European Parliament on the protection of animals used for scientific purposes and the German animal use and care laws (Versuchstierschutzgesetz) and approved by the German state administration Saxony-Anhalt (Projekt/AZ:42502-3-600-MLU).

\section{Whole-mount in situ hybridization (Wmish)}

Wmish was performed as before. ${ }^{16}$ Antisense digoxigeninlabeled UTP (Hoffman-La Roche Ltd., Basel, Switzerland) RNA probes were transcribed from plasmids: ami/ pBSKII(EcoRl/T7) and er71/pCS2+(EcoRI/T7). Chromogenic reactions were carried out using 4-nitro blue tetrazolium chloride/5-bromo-4-chloro-3-indolyl-phosphate (HoffmanLa Roche Ltd.). Stained embryos were photographed using
SZX12 stereomicroscope (Olympus Corporation, Tokyo, Japan) and DFC7000T digital camera (Leica Microsystems, Wetzlar, Germany).

\section{OXYREG setup}

The 4-channel OXYREG apparatus was purchased from Loligo ${ }^{\circledR}$ Systems (Viborg, Denmark). The ADA and analog digital converter (ADC) modules were connected to each other with the provided cables. Solenoid valves were connected to their respective inlets in the ADA module. Furthermore, nitrogen inlet was connected to the solenoid valve. OxyCTRL software was run on the computer and checked that all oxygen probes are displaying current percentage oxygen saturation levels in the tanks. After ensuring all connections, the new experiment was started and desired percentage oxygen saturation levels were selected in the OxyCTRL software for each tank. The OXYREG system regulated the entry of nitrogen gas until the desired point. It is advisable to start the experiment $\sim 6-12$ hours prior to introducing embryos to ensure uniform distribution and saturation of gases in the buffer and to calibrate oxygen probes before first use.

\section{Calibration of oxygen probes}

All oxygen probes were calibrated before using them for the experiment. Oxygen probes were connected to the OXYREG device. A $500 \mathrm{~mL}$ glass beaker was filled with $0.1 \times$ Modified Barth's Solution (MBS) buffer. All the oxygen probes were placed in the buffer. Nitrogen gas was perfused into the buffer until the voltage reading in the OxyCTRL software for that specific probe stabilizes. This reading was selected as "low point", which represents $0 \%$ oxygen saturation. Atmospheric oxygen was perfused into the buffer until the voltage reading in the OxyCTRL software for that specific probe stabilizes. This reading was selected as "high point", which represents maximum $/ 100 \%$ oxygen saturation $\left(0.2845 \mathrm{mmol} / \mathrm{L}\right.$ at $\left.20^{\circ} \mathrm{C}\right)$. The same procedure was repeated with each oxygen probe. It was not necessary to calibrate oxygen probes before every experiment. These oxygen probes are robust. Therefore, once calibrated, they can be used until significant changes are observed in the percentage oxygen saturation reading.

\section{Hypoxia chambers setup}

It was important to maintain constant temperature throughout the experiment as percentage oxygen saturation varies with temperature..$^{15}$ Therefore, room temperature was maintained at $20^{\circ} \mathrm{C}$ and thermostat of the cooling plate (Laboratoires Humeau, La Chapelle-sur-Erdre, France), was turned on and set to $20^{\circ} \mathrm{C}$ (Figure $1 \mathrm{~A}$ ). 
To ensure that all tanks are free of dust particles, they were rinsed with distilled water. The tanks were filled with $2.5 \mathrm{~L}$ of $0.1 \times$ MBS buffer. This left sufficient space between the buffer and the lids to enable a constant air exchange. All tanks were placed on the cooling plate, as shown in Figure 1B. Different positions for the equipment inside the tank were tested and optimized for efficient mixing of gases and reducing disturbances to the embryos in the tank. Therefore, it was important that all parts in the tanks were placed at the correct positions. The glass beaker with a Petri dish on top was placed on the right side of the tank, close to the front. The oxygen probe was placed on the right side close to the back. The air-stone for nitrogen gas inlet was placed at maximum distance to the embryos in the left corner at the back of the tanks (Figure 1A).

The tank was first closed with the larger part of the lid simultaneously installing the oxygen probe. A stirrer RW 11 basic (IKA, Staufen, Germany), was introduced and ensured that the stirring blade was positioned close to the bottom of the tank. Finally, the rest of the tank was closed with the smaller part of the lid concurrently placing the air-stone in the chamber. Ensure that the air-stone touches the bottom of the tank. Stirrer was turned on and set to $\sim 100-150 \mathrm{rpm}$. Nitrogen inlet pressure was set to 2 bars $(=29 \mathrm{psi})$. Higher pressure resulted in uneven oxygen distribution, and the produced currents removed the embryos from the Petri dish. This was repeated in all other tanks. A control tank equipped in the same way without a lid was used as a normoxic control.

\section{Onset of hypoxia}

All buffers and experimental embryos were maintained at $20^{\circ} \mathrm{C}$. After the first cleavage, 500 perfectly cleaved two-cell stage embryos were transferred into a fresh Petri dish and incubated further. At different development stages, such as gastrulation, neurulation, early and late tailbud stages, and as required, 100 of these embryos were transferred into preequilibrated hypoxia tanks using the small opening in the lid. The embryos were examined periodically for mortality, and dead embryos were removed immediately to reduce bacterial growth and infection of healthy embryos. Several parameters, such as developmental stage, time, and number of dead embryos, were documented every few hours (usually every $4-8$ hours). Once all embryos had been removed from a chamber, regulation through the OxyCTRL software was finished to reduce nitrogen gas waste (for troubleshooting, refer Table 1).

\section{Experiment}

In previous experiments, we had shown that embryos that were incubated in buffer containing only $4 \%$ of the possible $100 \%$ oxygen saturation developed much slower and the development of the cardiovascular system was dramatically affected in these embryos, compared to embryos maintained under control conditions. ${ }^{12}$ In the experiments described here, we wanted to analyze whether the onset of hypoxia during development causes different effects.
A

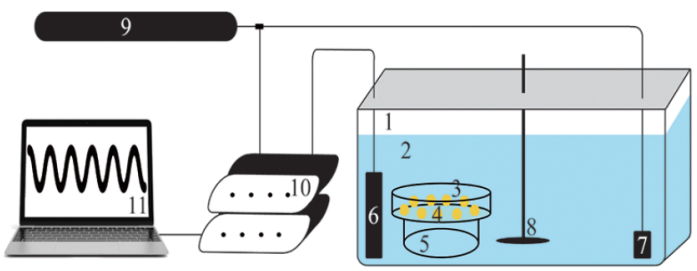

B

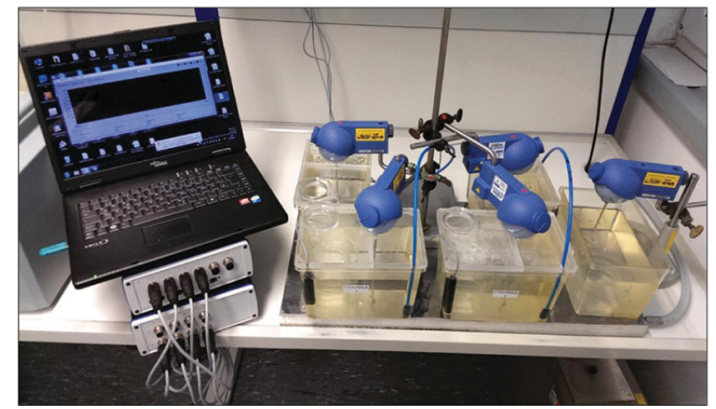

C

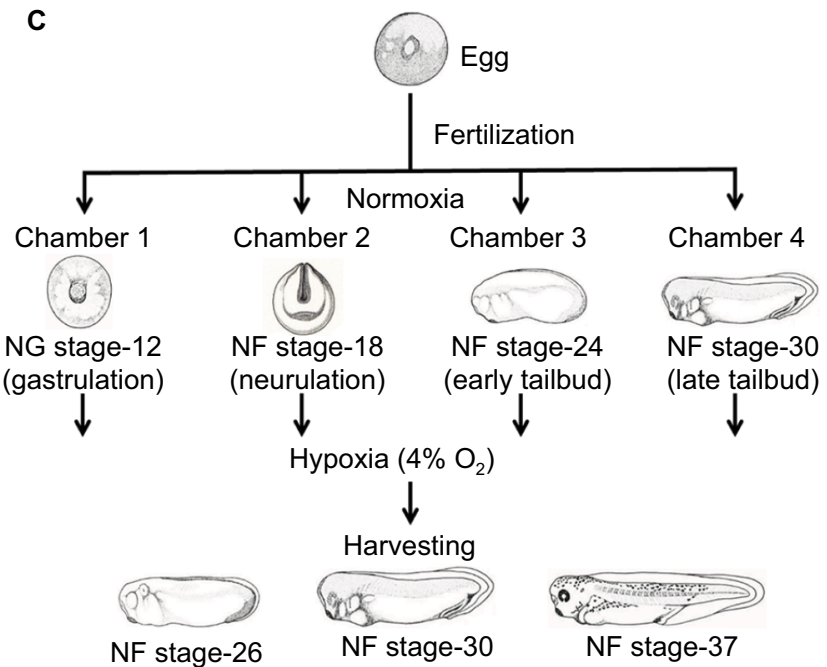

Figure I Experimental setup.

Notes: (A) Schematic representation of hypoxia chamber setup. I, plastic chamber; 2, $0.1 \times$ MBS buffer; 3, $10 \mathrm{~cm}$ Petri dish; 4, embryos; 5, glass beaker; 6, oxygen probe; 7, air-stone; 8, stirrer; 9, nitrogen gas supply; 10, OXYREG device; II, computer. (B) Running hypoxia experiment. Four hypoxia chambers were regulated at $4 \%$ oxygen along with a normoxia chamber. Temperatures in the chambers were maintained with the help of thermostat placed below the chambers. Regulation was computer controlled. (C) Growing embryos in hypoxia starting at various developmentally sensitive stages. Embryos were placed in hypoxia chambers at gastrulation, neurulation, and tailbud stages and harvested at a later point during development for assessment.

Abbreviation: MBS, Modified Barth's Solution. 
Table I Troubleshooting

\begin{tabular}{|c|c|c|}
\hline Issue & Possible reason & Solution \\
\hline OxyCTRL software not opening & Missing security key & Plug-in security key into the PC \\
\hline Faulty reading & Damaged membrane & Replace the membrane and electrolyte in the oxygen probe \\
\hline All solenoid valves not working & $\begin{array}{l}\text { ADA and ADC models are } \\
\text { turned off }\end{array}$ & $\begin{array}{l}\text { Turn on ADA and ADC module switches located at the back of each } \\
\text { module }\end{array}$ \\
\hline Stirrer not working & Stirrer rod was too close to the lid & Make sure that stirrer rod does not touch the edges of the lid \\
\hline Embryos can be found outside & Strong currents & Reduce stirrer rpm \\
\hline the Petri dish & High nitrogen gas inlet pressure & Reduce nitrogen gas pressure to the recommended level \\
\hline No effect on growth rate & Faulty oxygen probes & $\begin{array}{l}\text { Replace membrane, electrolyte, and calibrate oxygen probes. Replace if } \\
\text { necessary }\end{array}$ \\
\hline $\begin{array}{l}\text { Embryos in the hypoxia tanks } \\
\text { dying too early }\end{array}$ & Position of oxygen probe & $\begin{array}{l}\text { Make sure that the oxygen probe does not touch the bottom of the tank. } \\
\text { A gap of } \mathrm{I}-2 \mathrm{~cm} \text { should be maintained }\end{array}$ \\
\hline
\end{tabular}

Abbreviations: ADA, analog data acquisition; $A D C$, analog digital converter.

Hypoxia chambers were prepared as described earlier and started $\sim 15$ hours before the start of the experiment. All chambers were maintained at $4 \%$ oxygen saturation. Eggs were collected from female frogs and fertilized in vitro. After 1 hour, embryos were dejellied in 2\% cysteine ( $\mathrm{pH}$ 8.0) and washed four times with $0.1 \times$ MBS to wash out residual cysteine. Two-cell stage embryos were used in the experiment only, if $>95 \%$ of a clutch of eggs had been fertilized and simultaneously divided into two-cell stage embryos. Lower efficacy of fertilization may end in a higher variability of the phenotype. The experiments described here have been carried out at least twice. Embryos were examined periodically for proper development. Once embryos reached distinct developmental stages, eg, gastrulation (NF stage 12.5), neurulation (NF stage 18), early tailbud (NF stage 24), and late tailbud (NF stage 30), 100 embryos each were transferred into different chambers and incubated further. In addition, 100 embryos were transferred into a control chamber.

Once the embryos in the different hypoxia chambers reached NF stage 26, NF stage 30, and NF stage 35/36, a number of embryos, sufficient for further analysis, were removed from the chambers. These embryos were quickly fixed in $1 \times$ MEMFA in $5 \mathrm{~mL}$ glass tubes. Glass tubes were incubated at room temperature, rolling gently for 2 hours followed by two washes in $1 \times$ PBS and serial dehydration in ethanol ( 25 , 50,75 , and $100 \%$ ethanol, 5 min each). Embryos were then stored in $100 \%$ ethanol at $-20^{\circ} \mathrm{C}$. An oxygen regulation graph was plotted in Microsoft Excel by using the saved data from the OXYREG device (Figure 2A).

In general, the time to develop to NF stage $35 / 36$ was significantly increased independent of the onset of hypoxia. The developmental rate decreased as soon as the embryos were exposed to hypoxic conditions irrespective of the start- ing NF stage (Figure 2B). Embryos exposed to 4\% hypoxia commencing at NF stage 12.5 needed 1.6 times more to reach NF stage 35/36 compared to controls (65 hours for controls and 105 hours in 4\% hypoxia; Figure 2C). Starting hypoxia at later stages of development (NF stage 18, 24, and 30) also delayed development significantly. Interestingly, comparable developmental intervals were longer the earlier the embryos were exposed to hypoxia. For example, embryos transferred to the hypoxic tank at NF stage 12.5 needed 40 hours for the interval from NF stage 30 to NF stage 35/36, while embryos transferred to hypoxia at NF stage 30 needed only 24 hours to reach NF stage 35/36. Detailed developmental charts are listed in Table S1. The effect of the onset of hypoxia was most pronounced in embryos that were exposed to hypoxia starting at NF stage 12.5 and NF stage 24 . This suggests that gastrulation and tailbud stages are the most sensitive periods for the lack of sufficient oxygen. Detailed embryo viability is summarized in Table S2. To analyze the effect of hypoxia beginning at different stages of development on the vascular network, we monitored the expression of the endothelial precursor marker, er $71,{ }^{13}$ and of the endothelial differentiation marker, ami. ${ }^{16}$ In control embryos, er 71 was expressed in the posterior cardinal vein $(\mathrm{PCV})$, vitelline veins $(\mathrm{VV})$, ventral precursors (VP), posterior precursors (PP), and branchial arches regions (Figure 3A).

Expression of $\operatorname{er} 71$ was more pronounced at late tailbud stage in corresponding regions of the embryo (Figure 3B). In addition, er71-expressing cells (VV and VP) migrated away from the precursor pool at the ventral side of the embryo. Only little changes in er 71 expression were observed in early tailbud embryos, which were raised under hypoxia starting at gastrulation (NF stage 12; Figure 3C) and after neural tube closure (NF stage 18; Figure 3E). In general, er71 expression appeared less affected the later hypoxia treatment was 
A
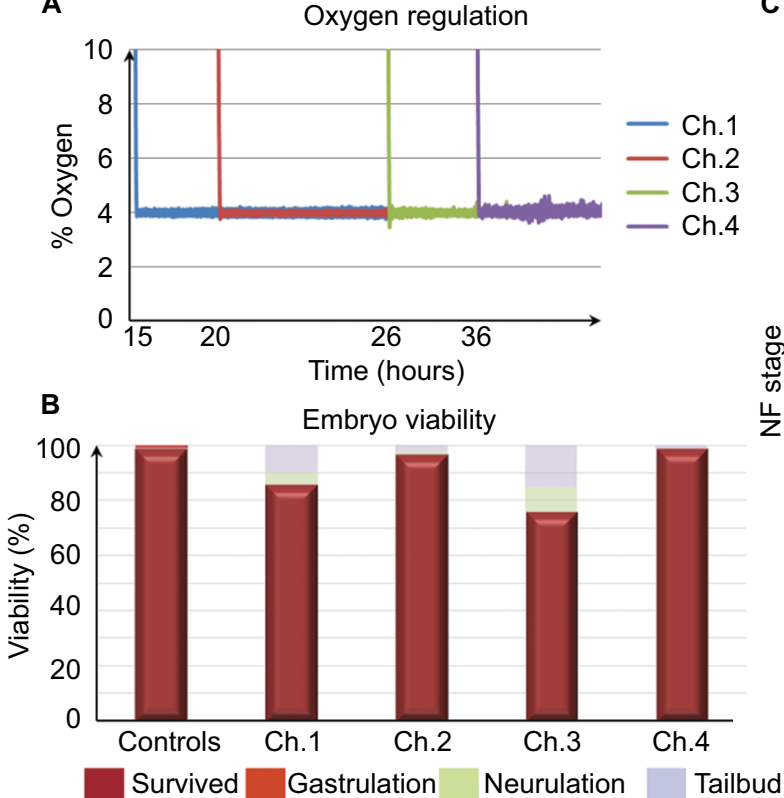

C

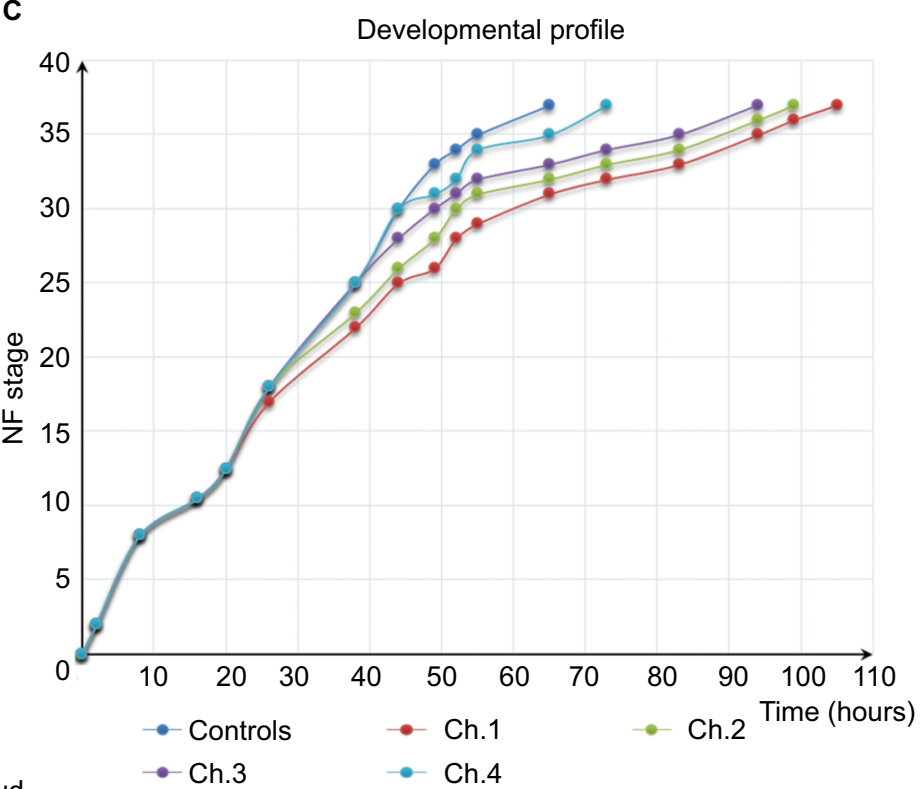

Figure 2 Hypoxia-sensitive period of early development.

Notes: (A) Oxygen regulation. The graph shows the regulated oxygen levels in four hypoxia chambers, which were regulated at $4 \%$ oxygen throughout the experiment. (B) Embryo viability. The bar graph shows the number of embryos that survived and died at various developmental stages throughout the experiment. (C) Developmental profile. The graph shows the time required for the development of embryos grown in four hypoxia chambers compared to normoxia embryos. Note the developmental delay starting from the point of exposure to hypoxic conditions in different chambers.

Abbreviations: Ch, channel; NF, Nieuwkoop \& Faber.

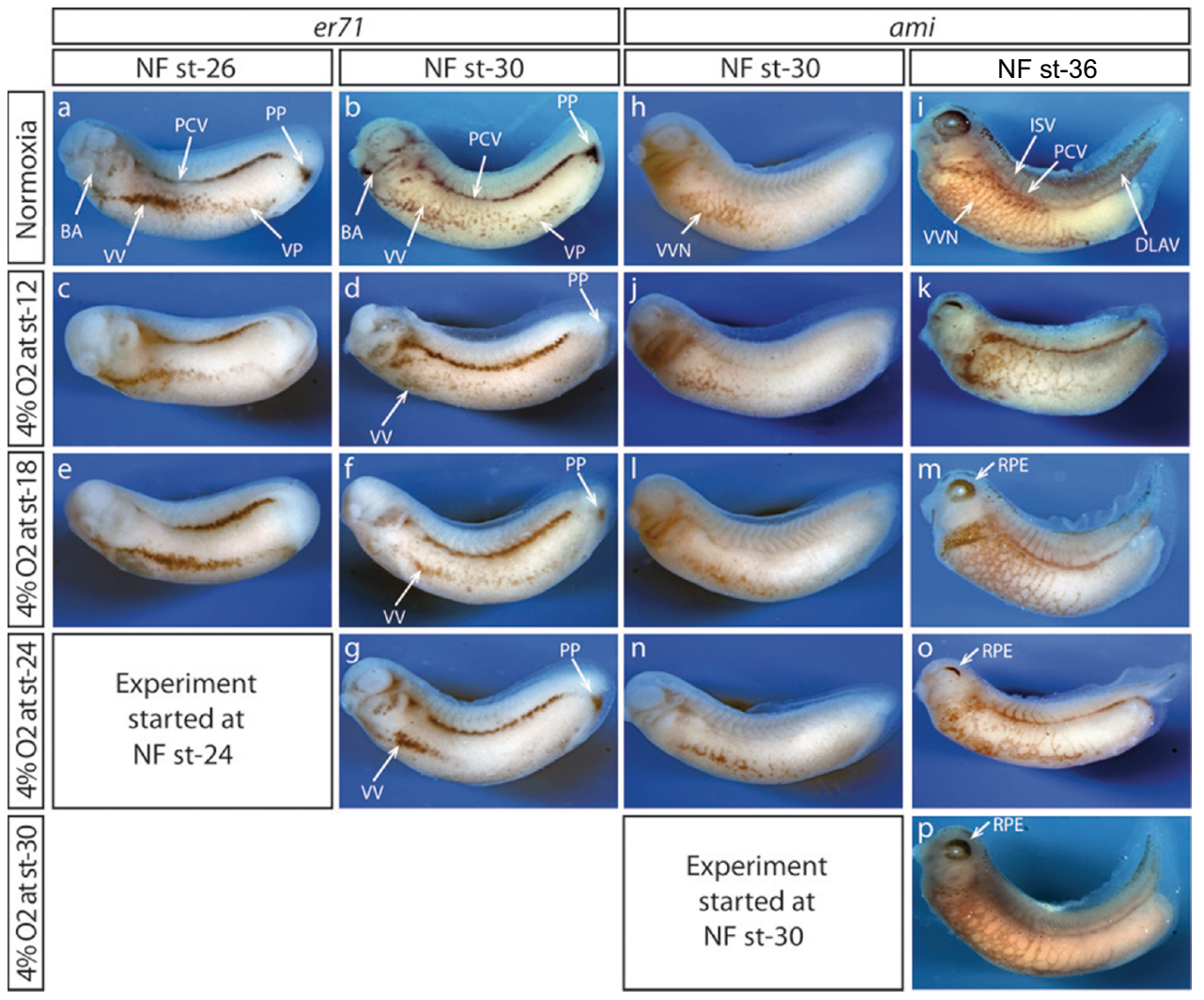

Figure 3 Hypoxia affects vascular network formation.

Notes: Wmish of embryos raised in hypoxia against endothelial precursors (er7l) and vascular network (ami). Even though embryos were exposed to $4 \%$ oxygen starting at different developmental stages, expression of endothelial precursor marker (er7I) and differentiation marker (ami) were strongly affected in all conditions. Reduction of er7I expression in PCV, VV, VP, PP and BA regions was observed in embryos exposed to 4\% oxygen at NF st-26 (a, c, e) and NF st-30 (b, d, f, g). Likewise, expression of ami was also reduced in VVN, ISV and DLAV regions at NF st-30 (h, j, l, n) and NF st-36 (i, k, m, n, o, p). In addition, impaired RPE was also observed (i, k, m, o, p).

Abbreviations: BA, branchial arches; DLAV, dorsal longitudinal anastomosing vessel; ISV, inter somatic vessels; NF, Nieuwkoop \& Faber; PCV, posterior cardinal vein; PP, posterior precursors; RPE, retinal pigment epithelium; st, stage; VP, ventral precursors; VV, vitelline veins; VVN, vascular vitelline network; Wmish, whole mount in situ hybridization. 
started. Accordingly, expression of er 71 was completely lost only in the PP of embryos starting hypoxia at NF stage 12 (Figure 3D, F, and G). In contrast, the ventral vein precursor cells (VV + VP) appeared more compact compared to the control the later the hypoxia treatment started, particularly at early tailbud stage (Figure 3B, D, F, and G).

The expression of $a m i$ was similarly altered. In embryos raised under normoxia, ami was expressed in the vascular vitelline network, inter somatic vessels (ISV), PCV, and dorsal longitudinal anastomosing vessel (DLAV) in late tailbud (NF stage 30) and in swimming tadpoles (NF stage 37; Figure $3 \mathrm{H}$ and I). The most severe effects on the formation of embryonic vessels were observed for the DLAV and ISV, which are missing upon early onset of hypoxia. Moreover, the branching of the VV was diminished, though the remaining ami-positive cells were still distributed along the whole ventral side. It is interesting to note that early hypoxia also suppressed the elongation of the tail (Figure 3K). In general, the later hypoxic treatment started, the better the ISV and DLAV were formed, and the branching of the VV was only little reduced compared to normal embryos (Figure 3C-P). Interestingly, the expression of ami in embryos that had been cultured under hypoxia starting at early tailbud stage confirmed our previously described observations. ${ }^{12}$ The retinal pigment epithelium formation of the eye was impaired, and only few of the arrested VV precursor cells that differentiated to $\operatorname{er} 71$ expressing precursor cells in the ventral region (Figure 3G) had been able to migrate away from their ventral location and instead differentiated into endothelial cells at their primary position (Figure 30). The findings had been confirmed in a second set of experiments. Thus, we could define at least two time points during development (gastrula and early tadpole stage), which were more sensitive for insufficient oxygen supply.

For the complete list of materials see Box S1.

\section{Discussion}

The principal aim of this study was to establish a protocol facilitating the culture of embryos in large numbers at controlled oxygen levels. This simple and efficient protocol allowed culturing of hundreds of Xenopus embryos in multiple tanks at desired oxygen levels. Analogously, this protocol can be adapted to culture embryos of other aquatic animals such as killifish, mussels, and blue crab with little to no modifications. The foremost advantage of this system was its ability to culture large number of embryos at different oxygen conditions simultaneously, which allows the generation of statistically significant data in a short period of time.
Another aim of this study was to investigate the sensitivity of particular embryonic stages to hypoxia during early development as an example for the use of the system. To address this question, we cultured embryos under hypoxia starting at different developmental phases. After testing various oxygen levels, $4 \%$ oxygen was chosen for this study as this was the lowest oxygen concentration, which allowed the development of swimming tadpoles. Culturing embryos in $2 \%$ oxygen leads to severe developmental impairment and premature death of large number of embryos before the early tailbud stages of development (data not shown). Interestingly, very similar effect on the developmental speed had been described previously for Xenopus embryos cultured in hypoxia chambers at 5,10 , and $21 \%$ air oxygen, which corresponds to $\sim 7.8,15.6$, and $32.6 \%$ oxygen in our setup. ${ }^{17}$ The embryos in the hypoxia chamber were cultured starting at gastrula stage, which was found to be most sensitive toward abrupt hypoxia. This might explain that we were able to use lower oxygen concentration in our setup.

We observed that gastrulation was a key check point during development, which was quite sensitive to the surrounding oxygen levels. This was supported by the observation that the embryos raised in $4 \%$ oxygen starting at gastrulation (channel [Ch].1) took a total of 105 hours to reach NF stage 35/36 in contrast to controls and embryos starting hypoxia at later stages of development: in ch.2 at NF stage 18 , in ch. 3 at NF stage 24 , and in ch. 4 at NF stage 30 , which reached the same stage in 99, 94, and 73 hours, respectively. We also observed that the longer the embryos were under hypoxia, the slower they developed. This was supported by the time taken by the embryos raised in ch.1 and ch.4. Embryos in ch. 1 took 40 hours to develop from NF stage 30 to NF stage 35/36, in contrast to embryos raised in $4 \%$ oxygen starting at late tailbud stage (ch.4), which took just 24 hours. This could be because the longer the embryos were under normoxia, the better they were able to cope with the reduced environmental oxygen. In addition, we observed significant death of embryos in ch. 3 compared to others. This observation was interesting, as the viability appears reduced compared to embryos in other chambers, although embryos in all chambers go through this phase. We hypothesize that this could be because of the beginning of organogenesis during early tailbud phase and an increased requirement of oxygen for these processes. It remains to be investigated further.

Performing Wmish on the embryos raised in different conditions to visualize vascular progenitors, and formation of vascular network also suggested that gastrulation was a key check point affecting not only developmental rate but 
also organ formation. It was obvious that the differentiation of endothelial progenitors was affected in the embryos raised in hypoxia regardless of the developmental stage when exposure to hypoxia started. Gastrulation could also be the key check point for vasculogenesis similar to the developmental rate as the strongest effect was seen in embryos that started to be exposed to hypoxia at NF stage 12. Although not as strong, er71-expressing cells were also reduced in embryos raised in hypoxia at later phases, suggesting that the onset of hypoxia during embryogenesis was always detrimental to development. As shown in our previous publication, the embryo can adapt to low oxygen concentrations by slowing down developmental speed and probably allows activating alternative energy-producing pathways. However, time points may exist when the need for energy was higher and the time to adapt to low oxygen levels takes too long for the energy need of a specific developmental step.

\section{Conclusion}

The establishment of a computerized, multi-way hypoxia system allowed us to raise hundreds of Xenopus embryos in one experiment at four different oxygen levels. In addition, we conclude from our experiments that gastrula and early tailbud embryos are most sensitive with regard to oxygen availability. It will be interesting to investigate oxygen consumption and therefore the need for energy changes during embryogenesis.

\section{Disclosure}

The authors report no conflicts of interest in this work.

\section{References}

1. Panchanathan R, Liu H, Choubey D. Hypoxia primes human normal prostate epithelial cells and cancer cell lines for the NLRP3 and AIM2 inflammasome activation. Oncotarget. 2016;7(19):28183-28194.
2. Glunde K, Shah T, Winnard PT Jr, et al. Hypoxia regulates choline kinase expression through hypoxia-inducible factor-1 alpha signaling in a human prostate cancer model. Cancer Res. 2008;68(1):172-180.

3. Hales CA, Kradin RL, Brandstetter RD, Zhu YJ. Impairment of hypoxic pulmonary artery remodeling by heparin in mice. Am Rev Respir Dis. 1983;128(4):747-751.

4. Dohlen G, Carlsen H, Blomhoff R, Thaulow E, Saugstad OD. Reoxygenation of hypoxic mice with $100 \%$ oxygen induces brain nuclear factor-kappa B. Pediatr Res. 2005;58(5):941-945.

5. Cordero GA, Karnatz ML, Svendsen JC, Gangloff EJ. Effects of lowoxygen conditions on embryo growth in the painted turtle, Chrysemys picta. Integr Zool. 2017;12(2):148-156.

6. Sui Y, Kong H, Shang Y, et al. Effects of short-term hypoxia and seawater acidification on hemocyte responses of the mussel Mytilus coruscus. Mar Pollut Bull. 2016;108(1-2):46-52.

7. Du SN, Mahalingam S, Borowiec BG, Scott GR. Mitochondrial physiology and reactive oxygen species production are altered by hypoxia acclimation in killifish (Fundulus heteroclitus). J Exp Biol. 2016;219(pt 8):1130-1138.

8. Brill RW, Bushnell PG, Elton TA, Small HJ. The ability of blue crab (Callinectes sapidus, Rathbun 1886) to sustain aerobic metabolism during hypoxia. J Exp Mar Bio Ecol. 2015;471:126-136.

9. Masamoto K, Takuwa H, Tomita Y, et al. Hypoxia-induced cerebral angiogenesis in mouse cortex with two-photon microscopy. Adv Exp Med Biol. 2013;789:15-20.

10. Cao R, Jensen LD, Soll I, Hauptmann G, Cao Y. Hypoxia-induced retinal angiogenesis in zebrafish as a model to study retinopathy. PLoS One. 2008;3(7):e2748.

11. Cao Z, Jensen LD, Rouhi P, et al. Hypoxia-induced retinopathy model in adult zebrafish. Nat Protoc. 2010;5(12):1903-1910.

12. Metikala S, Neuhaus H, Hollemann T. Suppression of vascular network formation by chronic hypoxia and prolyl-hydroxylase 2 (phd2) deficiency during vertebrate development. Angiogenesis. 2016;19(2): 119-131.

13. Hollemann T, Chen Y, Grunz H, Pieler T. Regionalized metabolic activity establishes boundaries of retinoic acid signalling. EMBO J. 1998;17(24):7361-7372.

14. Nieuwkoop PD, Faber J. Normal Table of Xenopus laevis (Daudin). North Holland, Amsterdam: Garland Publishing; 1967.

15. Wetzel RG. Limnology: Lake and River Ecosystems. San Diego: Academic Press; 2001.

16. Inui M, Asashima M. A novel gene, Ami is expressed in vascular tissue in Xenopus laevis. Gene Expr Patterns. 2006;6(6):613-619.

17. Hidalgo M, Le Bouffant R, Bello V, et al. The translational repressor 4E-BP mediates hypoxia-induced defects in myotome cells. J Cell Sci. 2012;125(pt 17):3989-4000. 


\section{Supplementary materials}

Table SI Developmental profiles

\begin{tabular}{|c|c|c|c|c|c|}
\hline Time (hours) & Controls & Ch.I & Ch.2 & Ch.3 & Ch.4 \\
\hline 0 & 0 & 0 & 0 & 0 & 0 \\
\hline 2 & 2 & 2 & 2 & 2 & 2 \\
\hline 8 & 8 & 8 & 8 & 8 & 8 \\
\hline 16 & 10.5 & 10.5 & 10.5 & 10.5 & 10.5 \\
\hline 20 & 12.5 & 12.5 & 12.5 & 12.5 & 12.5 \\
\hline 26 & 18 & 17 & 18 & 18 & 18 \\
\hline 38 & 25 & 22 & 23 & 25 & 25 \\
\hline 44 & 30 & 25 & 26 & 28 & 30 \\
\hline 49 & 33 & 26 & 28 & 30 & 31 \\
\hline 52 & 34 & 28 & 30 & 31 & 32 \\
\hline 55 & 35 & 29 & 31 & 32 & 34 \\
\hline 65 & 36 & 31 & 32 & 33 & 35 \\
\hline 73 & & 32 & 33 & 34 & 36 \\
\hline 83 & & 33 & 34 & 35 & \\
\hline 94 & & 34 & 35 & 36 & \\
\hline 99 & & 35 & 36 & & \\
\hline 105 & & 36 & & & \\
\hline
\end{tabular}

Notes: The table shows the time taken by the embryos grown in $4 \%$ oxygen starting at different developmental stages. Abbreviation: Ch, channel.

Table S2 Embryo viability

\begin{tabular}{|c|c|c|c|c|c|}
\hline NF stage & Controls & $4 \% O_{2} @$ stage 12 & 4\% O @ stage 18 & 4\% O @ stage 24 & $4 \% O_{2} @$ stage-30 \\
\hline 26 & 40 & 30 & 30 & 0 & 0 \\
\hline 30 & 30 & 30 & 30 & 40 & 0 \\
\hline 36 & 29 & 26 & 37 & 36 & 99 \\
\hline Survived & 99 & 86 & 97 & 76 & 99 \\
\hline Gastrulation & 1 & 0 & 0 & 0 & 0 \\
\hline Neurulation & 0 & 4 & I & 9 & 0 \\
\hline Tailbud & 0 & 10 & 2 & 15 & 1 \\
\hline
\end{tabular}

Notes: The table shows the total number of embryos collected at NF stage 26, 30, and 36. In addition, embryos that died during different developmental stages can be seen. Abbreviation: NF, Nieuwkoop \& Faber.

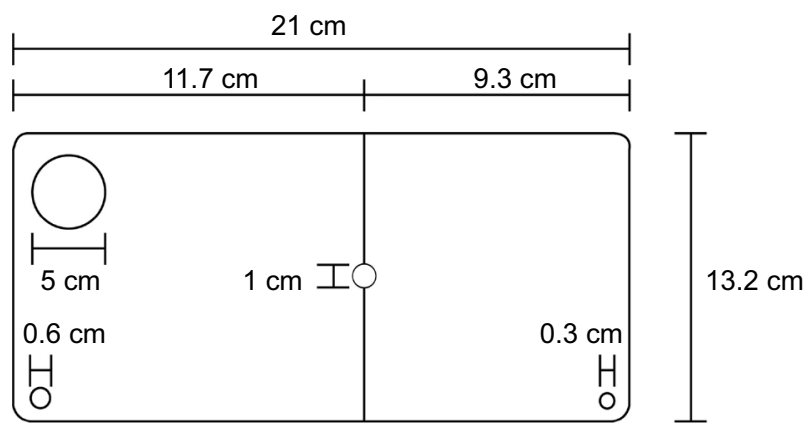

Figure SI The illustration showing the dimensions of the home-made lid for the hypoxia tanks. 
Box SI Reagents and materials

\section{Materials \\ Reagents}

The following reagents were used:

$0.1 \times$ MBS buffer,

$2 \%$ cysteine hydrochloride $(\mathrm{pH} 8.0)$,

I $\times$ MEMFA,

IXPBS,

Ethanol.

Equipment

The following equipment was used:

$10 \mathrm{~cm}$ Petri dishes,

$5 \times$ glass beakers $(5 \mathrm{~cm} \times 5 \mathrm{~cm} \times 5 \mathrm{~cm})$,

$5 \mathrm{~mL}$ glass vials,

$5 \times$ air-stone (fine pore size),

Tubing,

$5 \times 3 \mathrm{~L}$ plastic tanks $(22 \mathrm{~cm} \times 15 \mathrm{~cm} \times 13 \mathrm{~cm})$,

$4 \times$ custom plastic lids $(22 \mathrm{~cm} \times 15 \mathrm{~cm}$ ) made of three parts (refer

to Figure $S I$ for design details),

5X stirrers (RW II Basic; IKA, Staufen, Germany),

Stands to hold stirrers in place,

Custom-made cooling plate cooled by a thermostat (Laboratoires

Humeau, La Chapelle-sur-Erdre, France),

Loligo ${ }^{\circledR}$ Systems 4-channel oxygen regulator (Viborg, Denmark),

$4 \times$ solenoid valves,

$4 \times$ oxygen probes,

Analog data acquisition (ADA) module,

Analog digital conversion (ADC) module,

Connecting wires to connect two modules,

OxyCTRL software (Version 1.0.0.0 released on November 2I,

20II),

Secure key,

PC running Microsoft Windows 7 OS,

Microsoft Office 2013,

Pasteur pipettes.

\section{Hypoxia}

\section{Publish your work in this journal}

Hypoxia is an international, peer-reviewed, open access journal that aims to improve understanding of the biological response to hypoxia. The journal will publish original research articles, reviews, methodological advances, clinical studies, and expert opinions that identify developments in the regulation of the physiological and pathological responses to

Submit your manuscript here: https://www.dovepress.com/hypoxia-journal

\section{Dovepress}

hypoxia and in the therapeutic targeting of hypoxia-responsive pathways. The manuscript management system is completely online and includes a very quick and fair peer-review system, which is all easy to use. Visit $\mathrm{http}: / / \mathrm{www}$. dovepress.com/testimonials.php to read real quotes from published authors. 